\title{
The Influence of Temporary Blockage of Semicircular Canals on the Loss and Recovery of Vestibuloocular Reflexes
}

\author{
N. H. Barmack \\ Department of Ophthalmology, Neurological Sciences Institute, Good Samaritan Hospital and Medical Center, Portland, \\ Oregon 97209
}

\begin{abstract}
The efficacy of the technique of semicircular canal plugging in selectively blocking the function of individual semicircular canals was evaluated in rabbits. The horizontal and vertical vestibuloocular reflexes (HVOR, VVOR) and the horizontal optokinetic reflex (HOKR) were measured before the horizontal or anterior semicircular canals (HSCs, ASCs) were plugged bilaterally with small spindles of silver wire. These reflexes were also measured after the plugs were removed. The gain of the HVOR was reduced to less than 0.05 at all frequencies tested after plugs were inserted into the HSCs. The gain of the HOKR was not influenced by the plugging operation. These data were interpreted to mean that the plugs blocked the normal fluid dynamics of the HSCs, but did not alter the spontaneous activity of HSC afferents. The gain of the VVOR was not reduced by plugs of the HSCs. The gain of the VVOR was reduced by plugs of the ASCs. The residual VVOR was attributed to stimulation of the utricular otoliths at lower frequencies and stimulation of the posterior semicircular canals at higher frequencies. The gain of the HVOR was reduced $10-40 \%$ by plugs of the ASCs. These data, in conjunction with the presence of a small residual gain of the HVOR following bilateral plugs of the HSCs, suggest that the ASCs contribute to the HVOR. The gains of the HVOR and VVOR recovered to within $88 \%$ of the preplug values once the plugs of the HSCs or ASCs were removed. However, the protracted time course of this recovery, 10$15 \mathrm{~d}$, indicated that the plugging operation, although reversible, does cause some temporary damage to the peripheral labyrinth.
\end{abstract}

The vestibular nuclei of mammals are the focal point for convergent sensory information used to regulate reflexive eye movements, as well as other postural reflexes. In addition to the projection of primary vestibular afferents, the vestibular nuclei receive visual, as well as neck, proprioceptive information (Henn et al., 1974; Keller and Daniels, 1975; Rubin et al., 1977). This sensory convergence is also reflected in the deficits in reflexive eye movements caused by damage to the vestibular apparatus. Bilateral labyrinthectomies not only abolish vestibuloocular reflexes, but also reduce the gain of optokinetic reflexes and shorten the duration of optokinetic afternystagmus in cats (Capps

\footnotetext{
Received June 15, 1987; revised Oct. 19, 1987; accepted Dec. 3, 1987.

This research was supported by Grant EY04167 and by a grant from the Oregon Lions Sight and Hearing Foundation.

Correspondence should be addressed to Neal H. Barmack, Ph.D., Neurological Sciences Institute, 1120 N.W. 20th Avenue, Portland, OR 97209.

Copyright (C) 1988 Society for Neuroscience 0270-6474/88/082816-11\$02.00/0
}

and Roth, 1978), rabbits (Collewijn, 1976; Barmack et al., 1980), monkeys (Cohen et al., 1973), and humans (Zee et al., 1976). There are 2 immediate consequences of labyrinthectomies or vestibular neurectomies on secondary vestibular neurons: (1) an obvious loss of modulation of secondary neurons by vestibular primary afferent signals, and (2) a decrease in spontaneous primary vestibular afferent input to secondary vestibular neurons, with a consequent reduction of spontaneous secondary vestibular neuronal activity (Precht et al., 1966). Following a unilateral labyrinthectomy, the loss of secondary vestibular neuronal activity ipsilateral to the damaged labyrinth creates an asymmetric output signal from the vestibular nuclei, and results in both nystagmus and postural imbalance (Dow, 1938; Precht et al., 1966; Fisch, 1973; Baarsma and Collewijn, 1975; Lacour et al., 1976; Jensen, 1979; Maioli et al., 1983). The decrease in spontaneous secondary vestibular neuron activity caused by bilateral labyrinthectomies restricts the discharge frequencies over which secondary vestibular neurons can respond to central optokinetic signals, and accounts for the reduction in gain of optokinetic reflexes (Cohen et al., 1973; Zee et al., 1976; Capps and Roth, 1978; Barmack et al., 1980).

The technique of semicircular canal plugging, mechanically blocking the membranous semicircular canals and thereby preventing changes in pressure across the cupula during angular acceleration of the head, offers the possibility of distinguishing between deficits in reflexive eye movements of vestibular origin and deficits that might be ascribed to a reduction in the spontaneous activity of secondary vestibular neurons. This technique has been used to study the contributions of individual semicircular canals to reflexive eye movements (Money and Scott, 1962; Baker et al., 1982; Paige, 1983; Bohmer et al., 1985). Plugs have been made by drilling through the bony labyrinth with a fine dental burr that presumably seals both the bony and membranous labyrinths with bone dust. The efficacy of this procedure in blocking semicircular canal function has been confirmed, although the demonstration of a limitation of the effects of canal plugging to only the plugged canal(s) has not been substantiated. The spontaneous activity of vestibular primary afferents that were presumed to innervate a plugged anterior semicircular canal (ASC) was not "obviously different" from the spontaneous activity of other primary afferents that innervated other presumed undamaged semicircular canals in the same labyrinth (Goldberg and Fernandez, 1975). These data were confirmed, using the same plugging method in squirrel monkeys subjected to a unilateral plugging operation (Paige, 1983). However, following a unilatcral plugging opcration, squirrcl monkeys evinced an immediate and persistent nystagmus, with the slow phase 
directed towards the plugged labyrinth (Paige, 1983). If the plugging operation caused no alteration in the spontaneous primary afferent activity, what was the origin of the nystagmus?

An alternative index of the efficacy of the semicircular canal plugging procedure in not modifying the spontaneous activity of primary vestibular afferents could be obtained from the measurement of the horizontal optokinetic reflex (HOKR) before and after the semicircular canals were plugged. The gain of the HOKR, but not of the vertical optokinetic reflex (VOKR), is reduced by the loss of primary vestibular activity caused by bilateral labyrinthectomies. At the same time, by plugging one pair of semicircular canals and measuring vestibuloocular reflexes that presumably should not be mediated by these canals, it would be possible to evaluate whether the effects of semicircular canal plugs are indeed restricted to the plugged canals.

The present experiment was undertaken with the purpose of testing whether the HOKR would be influenced by plugs of pairs of semicircular canals. A second aim of this experiment was to determine whether the effects of semicircular canal plugs, as measured by the HOKR, horizontal vestibuloocular reflexes (HVOR), and vertical vestibuloocular reflexes (VVOR) were restricted to a single reflex plane. A third aim was to determine whether the effects of semicircular canal plugs were reversible. The principal findings of this investigation are that (1) plugs of the semicircular canals do not significantly reduce the gain of the HOKR; (2) the ASCs, as well as the horizontal semicircular canals (HSCs), contribute to the HVOR; and (3) the effects of canal plugs are reversible. However, the protracted time course of recovery following removal of the plugs suggests that the plugging procedure reversibly damages the peripheral labyrinth.

\section{Materials and Methods}

Preparatory surgical procedures. Twenty-one pigmented rabbits, weighing $1.0-2.0 \mathrm{~kg}$, were the subjects in this experiment. Twelve rabbits received bilateral plugs of the HSCs and 7 rabbits received bilateral plugs of the ASCs. The plugs were left in place for $26-48 \mathrm{hr}$. Two rabbits received temporary bilateral plugs of the HSCs, which were left in place for only $1 \mathrm{~min}$. In preparatory operations, the rabbits were anesthetized with intramuscular injections of ketamine hydrochloride $(50 \mathrm{mg} / \mathrm{kg})$, xylazine $(6 \mathrm{mg} / \mathrm{kg})$, and acepromazine maleate $(1.2 \mathrm{mg} / \mathrm{kg})$. Each rabbit's head was aligned in a stereotaxic apparatus so that the lambda suture was $1.5 \mathrm{~mm}$ above the bregma suture. Two stainless steel screws (832 ) were anchored to the calvarium with 4 smaller, peripherally placed stainless steel screws (2-56) and dental cement. The 2 larger screws mated with a horizontal steel rod that restrained the rabbit's head during experiments and supported the eye position monitor. When fastened to the rate table, the rod was tilted in the posterior-anterior direction at an angle of $12^{\circ}$ in order to align the plane of the HSCs with the earth horizontal axis. The correct alignment of the HSCs with the earth horizontal axis was confirmed in 4 rabbits in which postmortem measurements of the planes of the each of the semicircular canals were made with the skull affixed to the head restraint rod. This method of head restraint left unobscured the central $100^{\circ}$ of arc of the visual fields of each eye.

Plugging operations of the semicircular canals. Rabbits were anesthetized with halothane or ketamine hydrochloride $(50 \mathrm{mg} / \mathrm{kg})$, xylazine $(6 \mathrm{mg} / \mathrm{kg})$, and acepromazine maleate $(1.2 \mathrm{mg} / \mathrm{kg})$. A preauricular approach was made to the middle ear (Suzuki et al., 1969). Under a dissecting microscope, the tympanic membrane and the malleus were removed, exposing the bony surfaces of the HSCs and ASCs. The stapes was left in place. For HSC plugs, further exposure of the bony canal was achieved by enlarging the middle ear cavity posterodorsally. For ASC plugs, further exposure of the bony canal was achieved by removing the incus, which inserts on a bony fin separating the ampullae of the HSC and ASC. The opening was then enlarged anterodorsally. After exposure of a particular semicircular canal, a small opening was made in the bony wall of the cand with a \#1 dental buir, leaving the membranous canal intact. A $0.075 \mathrm{~mm}$ silver wire was melted by a small flame into a blunt spindle approximately $0.5-1.5 \mathrm{~mm}$ in length and $0.150-0.200 \mathrm{~mm}$ in maximum diameter. The spindle was inserted into the opening of the bone, gently compressing the membranous canal. The spindle was left with its "tail" protruding from the bony opening so that it could be removed easily in a subsequent operation. The opening was partially sealed with bone wax to prevent leakage of perilymph from the bony defect. The approximate locations of the plugs of the HSC and ASC are illustrated in Figure 1. Typically the plugs were left in place $48 \mathrm{hr}$, after which they were removed while the rabbit was anesthetized with halothane. In preliminary experiments it was found that if the plugs were left in place for a longer duration, recovery was less likely to occur when the plugs were removed, and when it did occur, it was less complete. All eye movement recordings obtained from rabbits that had received bilateral semicircular canal plugs of $24-48 \mathrm{hr}$ duration were obtained at least $24 \mathrm{hr}$ after the plugs were inserted. The rabbits were maintained for 1 week with daily intramuscular injections of penicillin $\mathrm{G}(50,000 \mathrm{U} / \mathrm{kg})$ and prednisolone $(6 \mathrm{mg} / \mathrm{kg})$.

Eye position recording. Eye position was measured with an infrared light projection technique. A small suction cup bearing a light-emitting diode (LED) was attached to the eye, which was topically anesthetized with proparacaine hydrochloride. The LED projected a narrow beam of infrared light onto a photosensitive $\mathrm{X}-\mathrm{Y}$ position detector (SC-50; United Detector Technology) that was fixed relative to the head and located 2-4 mm from the tip of the LED (SSL-315; G.E.). The photosensitive surface gave a continuous $X-Y$ indication of the position of the incident centroid of infrared light. The transducer had a diameter of $57 \mathrm{~mm}$ and was mounted in an aluminum ring that had a fixed position relative to a supporting rod used to position the transducer in front of the eye. The transducer could be rotated $30^{\circ}$ within the ring to align the horizontal and vertical axes of the transducer with the horizontal and vertical axes of vestibularly evoked eye movements. The transducer and ring were inscribed with vernier markings, allowing axial adjustments of the transducer that were accurate to $0.2^{\circ}$. A null axis technique was used to align the transducer during vestibular stimulation. For measurements of the HVOR, the transducer was rotated until a minimum vertical eye movement was evoked, and for measurements of the VVOR, the axis of rotation was adjusted until stimulus-evoked horizontal eye movements were minimized. The eye movement transducer was calibrated by moving the LED on a model of the rabbit eye through a known angular displacement. This system has a sensitivity of $60 \mathrm{mV} / \mathrm{deg}$ and is linear to within $5 \%$ for eye deviations of $\pm 15^{\circ}$.

Vestibular stimulation. Rabbits were mounted with heads fixed at the center of rotation of a biaxial servocontrolled rate table. The body of the rabbit was encased in foam rubber and supported with elastic straps to a plastic tube aligned with the longitudinal axis. The rate table was oscillated sinusoidally at a constant amplitude of $\perp 10^{\circ}$ over a frequency range of $0.01-0.80 \mathrm{~Hz}$ about the earth vertical axis (evoking the HVOR) or about the earth horizontal axis (evoking the VVOR). Table position signals were obtained from servopotentiometers for each axis of rotation. During vestibular stimulation, the vision of both eyes of the rabbit was always completely occluded by 3 layers of black cloth.

Optokinetic stimulation. The rate table was located $55 \mathrm{~cm}$ from the center of a rear-projection tangent screen that subtended $70^{\circ} \times 70^{\circ}$ of visual angle. The optokinetic stimulus was rear-projected onto the screen by beaming the image projected by a $35 \mathrm{~mm}$ slide projector off 3 firstsurface mirrors, 2 of which were mounted orthogonally on EEG pen motors. Appropriate voltage ramps to the pen motors generated constant velocity movement along the horizontal and vertical axes of the projected image, a random-dot, contour-rich pattern. A rear-projection tangent screen is truly tangent at only one point on the optical axis. Consequently, a "constant velocity" target would have a lower angular velocity at the extreme edges of the screen. In the present experiment, the velocity of the optokinetic stimulus was about $12 \%$ lower for the most peripheral $5^{\circ}$ relative to the most central $5^{\circ}$ subtended by the $70^{\circ}$ tangent screen. The calibration for optokinetic stimulus velocity was based on the average velocity of a point in transversing the entire tangent screen. The rabbits were aligned with respect to the tangent screen so that the central $70^{\circ}$ of the left eye was stimulated while the rabbits were maintained in either the prone or supine orientation. The eye position of the unstimulated right eye was recorded.

Vestibular and optokinetic reflex testing. The gains $(G)$ of the HVORs and VVORs were determined from measurements of the peak eye velocities attained during each half-cycle of sinusoidal rotation. $G=\left(V_{\mathrm{R}}\right.$ $\left.+V_{\mathrm{t}}\right) / 2 V_{\mathrm{T}}$, where $V_{\mathrm{R}}$ - peak compensatory eye velocity to the right (or up), $V_{\mathrm{L}}=$ peak compensatory eye velocity to the left (or down), and $V_{\mathrm{T}}$ 


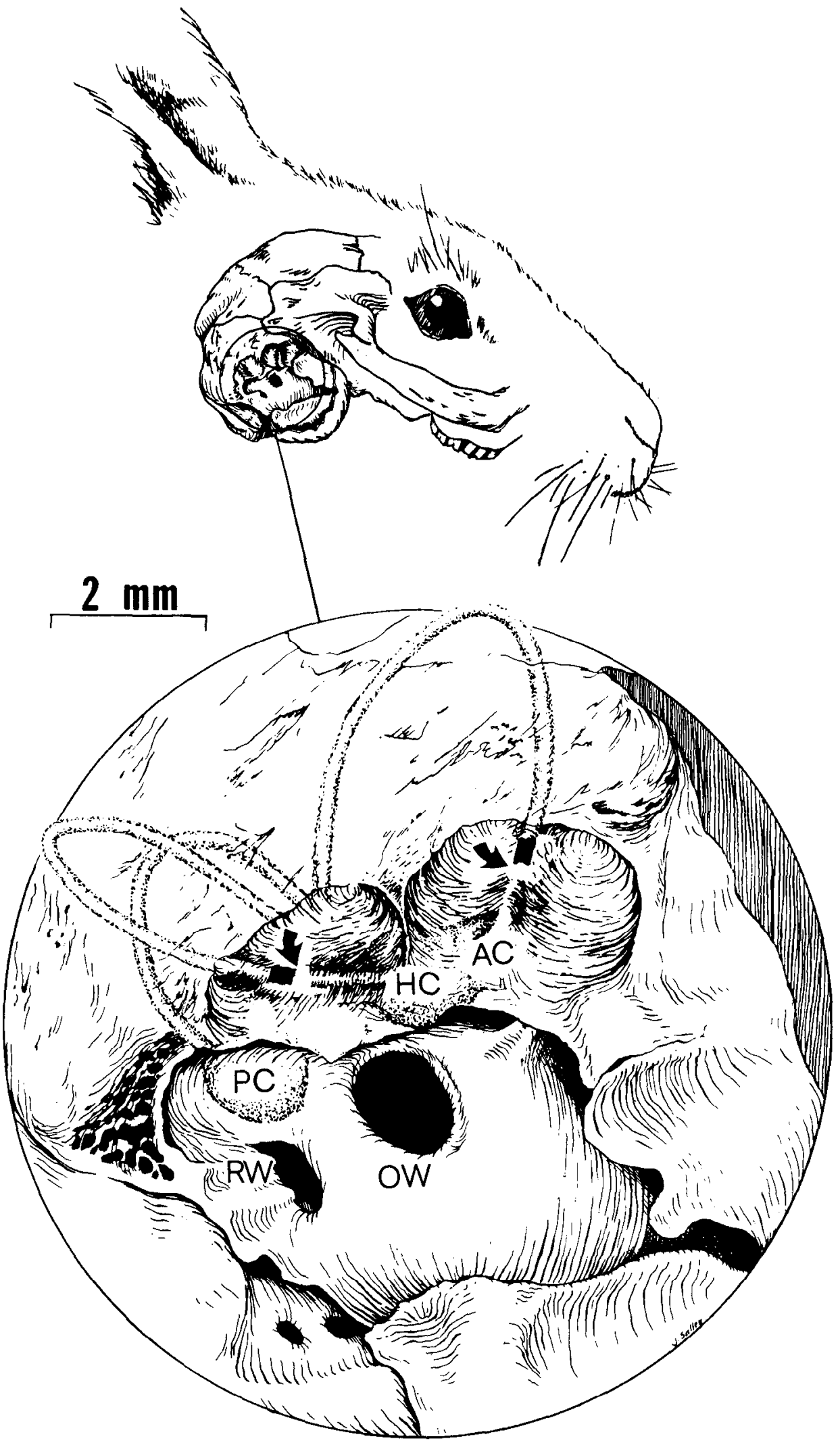

Figure 1. Illustration of the labyrinth of the rabbit. Arrows indicate where plugs were inserted into the horizontal or anterior semicircular canals. $A C, H C$, $P C$, ampullae of the anterior, horizontal, and posterior semicircular canals; $R W$, round window; $O W$, oval window.

position and table position signals (eye position $+180^{\circ}$ in relation to head position). Phase was measured at each half-cycle of rotation. At stimulation frequencies below $0.02 \mathrm{~Hz}, 2$ cycles of stimulation were usually measured. At frequencies above $0.04 \mathrm{~Hz}, 3$ or more cycles were usually measured.
The gain ( $G=$ eye velocity/stimulus velocity) of the HOKR was
$=$ peak table velocity. Eye and table velocity were measured by electronic differentiation of the eye and table position signals. The differentiator was calibrated with voltage ramps and was linear to within $2 \%$ of the range of eye and table velocities encompassed in this experiment. Because of possible distortions in phase introduced by electronic differentiation, the phases of the HVOR and VVOR were obtained from eye 
The Journal of Neuroscience, August $1988,8(8) 2819$
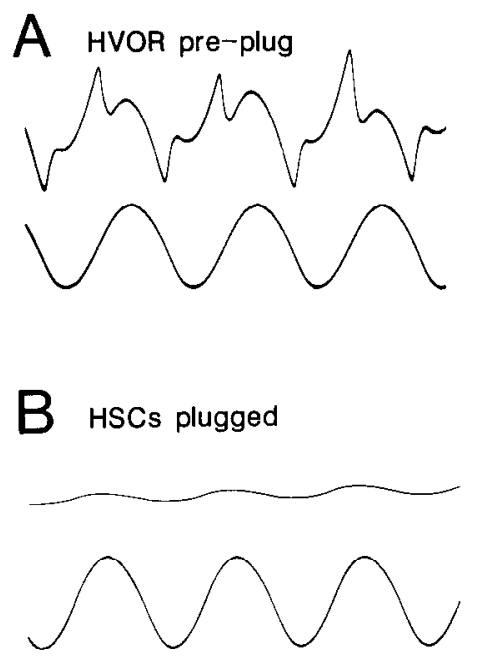

C 4 hours post-plug removal
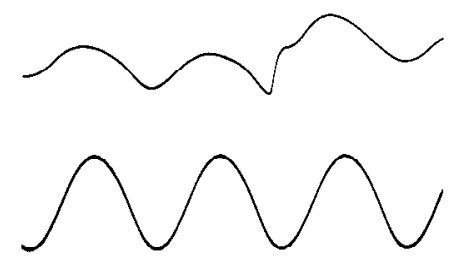

D 28 hours post-plug removal

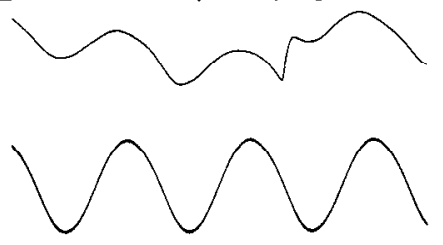

E 4 days post-plug removal
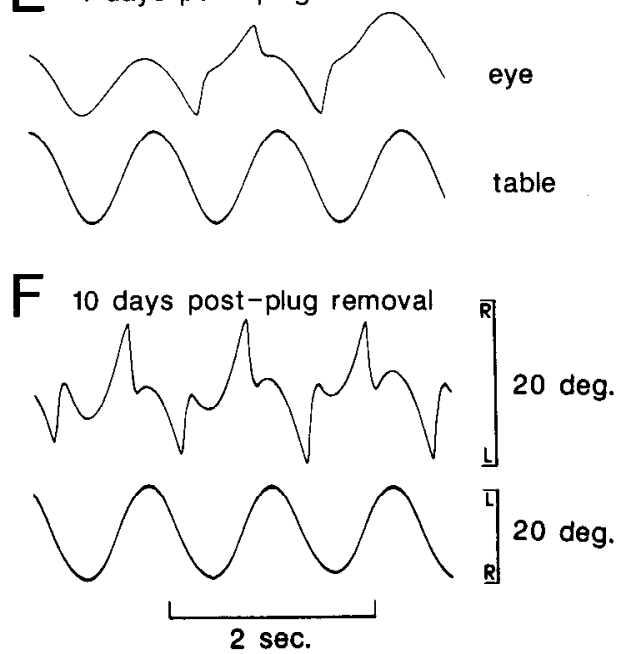

Figure 2. The horizontal vestibuloocular reflex (HVOR) evoked before and after bilateral plugs of the horizontal semicircular canals (HSC). Vestibular stimulation was given at $0.8 \mathrm{~Hz}, 20^{\circ}$ amplitude before the HSCs were plugged $(A), 24 \mathrm{hr}$ after the plugs were inserted $(B)$, and at various times after the plugs were removed $(C-F)$. determined from measurements of the peak eye velocities attained during constant velocity optokinetic stimulation in the posterior-anterior direction of the left eye. Typically, optokinetic reflex testing began at low stimulus velocities, which were slowly and continuously incremented to higher stimulus velocities. All data were recorded on an FM tape recorder, and subsequently displayed on a storage oscilloscope for measurement and analysis.

Histological analysis of plugged canals. The ampullae of 2 rabbits with unilaterally plugged HSCs were examined histologically. In one of these animals, the plug was left in place for $48 \mathrm{hr}$. In the other animal the unilateral plug was left in place for $30 \mathrm{~d}$. The bony labyrinths were decalcified in EDTA (Seilly, 1982), dehydrated, embedded in low-viscosity epoxy resin (Spurr), cut into semithin sections $(1-5 \mu \mathrm{m})$, and stained with hemotoxylin and eosin.

\section{Results}

\section{Behavioral characteristics of rabbits with plugged semicircular} canals

The immediate postoperative appearance of rabbits with bilaterally plugged HSCs was characterized by horizontal oscillations of the head of increasing amplitude when movements were attempted or if the head was externally perturbed. For rabbits with plugs of the ASCs, these head oscillations were rolling movements about the longitudinal axis. The oscillations abated even before the plugs were removed, and recovery of semicircular canal function took place. If the plugging operation was performed successfully, there was no development of spontaneous nystagmus. However, if the membranous labyrinth was ruptured or even abraded when the plugs were inserted, then not only did a spontaneous nystagmus develop, but the effects of the plugs were irreversible and progressive loss of other semicircular canal and otolith function inevitably ensued. A unilateral loss of otolith function was indicated by a progressive head tilt toward the damaged side. Once it developed, this head tilt was permanent.

\section{Effects of bilateral plugs of the horizontal semicircular canals on the horizontal and vertical vestibuloocular reflexes}

Bilateral plugs were placed in the HSCs of 5 rabbits. They were left in place for $48 \mathrm{hr}$. For each rabbit, measurements of the HVOR, VVOR, and HOKR were made (1) before the plugs were inserted, (2) while the plugs were in place, and (3) after the plugs were removed. The HSC plugging operation caused a marked decrement in the gain of the HVOR at all frequencies tested, but did not totally abolish the HVOR at stimlus frequencies greater than $0.10 \mathrm{~Hz}$ (Figs. 2, 3). The HVOR at these higher stimulus frequencies was characterized by a low gain (less than 0.05 at $0.8 \mathrm{~Hz}$ ) and a large increase in phase lead of $45^{\circ}$ at $0.8 \mathrm{~Hz}$ (eye position in relation to table position $+180^{\circ}$ ) (Fig. 3 ). In addition to the changes in gain and phase, the bilateral plugs of the HSCs also caused a change in the trajectory of vestibularly evoked eye movements. Before the plugs were inserted into the HSCs, the axes of eye movements evoked by vertical and horizontal stimulation were nearly orthogonal, having an angle of $88.6^{\circ}$. Immediately after the HSCs were plugged, the relative angle of the eye movements evoked by horizontal and vertical vestibular stimulation changed to $76.0^{\circ}$ (Fig. 4). This deviation in axial alignment was measured at stimulus frequencies of $0.4 \mathrm{~Hz}$ for both the HVOR and the VVOR. When the rate table was rotated to the left, evoking a rightward movement of the right eye, the movement also had a downward vertical component. When the rate table was rotated about the longitudinal axis onto the left side, then the evoked downward movement of the right eye had a rightward component. Two weeks after the removal of the plugs, the gain and phase of the HVOR, as well as the axial alignment of the HVOR and VVOR, returned to near the preplug values (Figs. 3, 4). The horizontal and vertical axes of the eye movement monitor were coaxial, 
Figure 3. Influence of bilateral plugs of the HSCs on the HVOR. The HVOR of 5 rabbits was tested before (filled circles), 24-48 $\mathrm{hr}$ after (open circles) the HSCs were plugged, and 2-3 weeks after the plugs were removed (filled triangles). One SD is illustrated for each data point for this and subsequent figures.
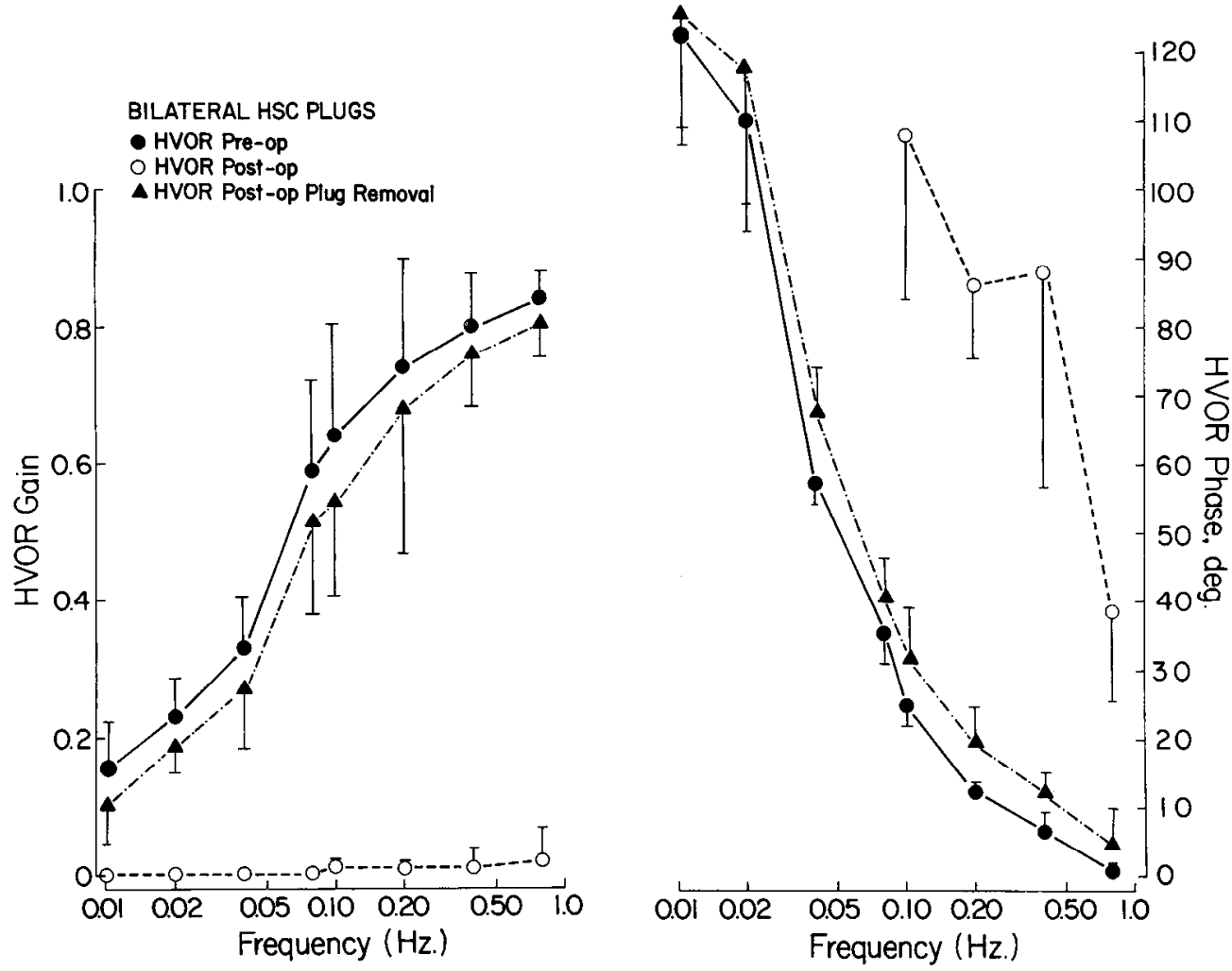

with the planes of rotation of the rate table at $00.0^{\circ}$ on the eyetransducer vernier setting (Fig. 4). But the axes of the horizontal and vertical semicircular canals could have been slightly misaligned with respect to the axes of rotation, since the alignment

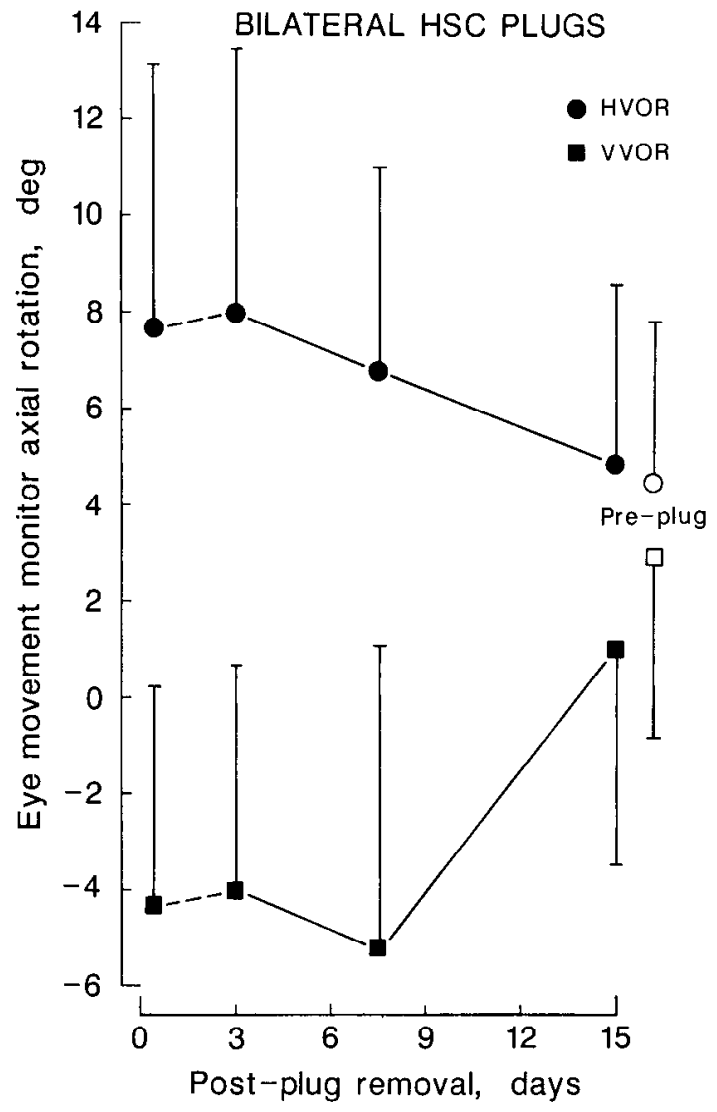

of the head in the apparatus was based on measurements of external bony landmarks of the HSCs. This may explain why the "preplug" and "rccovercd" valucs of axial alignment for the HVOR and VVOR converged at the absolute value of $4^{\circ}$ (Fig. 4). However, an error of this magnitude would lead to an underestimate of the gains of all vestibuloocular reflex measurements of less than $1 \%$.

The VVOR was measured before and 24-48 hr after plugs were inserted into the HSCs. The gain and phase of the VVOR were not influenced by the HSC plugging operation (Fig. 5).

\section{Effects of bilateral plugs of the horizontal semicircular canals on the horizontal optokinetic reflex}

The HOKR was measured before and $24-48 \mathrm{hr}$ after plugs were inserted into the HSCs. This reflex was evoked by monocular, posterior-anterior, optokinetic stimulation of the left eye over a range of stimulus velocities of $0.25-50.00 \mathrm{deg} / \mathrm{sec}$. Visual stimulation of the right eye was prevented by covering the eye and the eye movement transducer with 3 layers of black cloth. The gain of the HOKR was near the preplug values for all velocities tested. These values may be compared with the gain of the monocular HOKR, recorded under identical conditions in a

Figure 4. Changes in the axes of eye movements caused by bilateral plugs of the HSCs. The alignment axes for the HVOR (circles) and $0.4 \mathrm{~Hz}$ before (open symbols), $24 \mathrm{hr}$ after (solid symbols connected by dashed line to other data points) bilateral plugs of the HSCs were inserted, and at different times after the plugs were removed. If the axes of eye movements evoked by the horizontal and vertical vestibular stimulation were perfectly orthogonal, then axial alignment for the eye movement monitor would be the same for the HVOR and VVOR. Bilateral plugs of the HSCs caused the right eye to move down when it moved to the right, and to the right when it moved down. VVOR (squares) were measured in 5 rabbits at stimulus frequencies of 


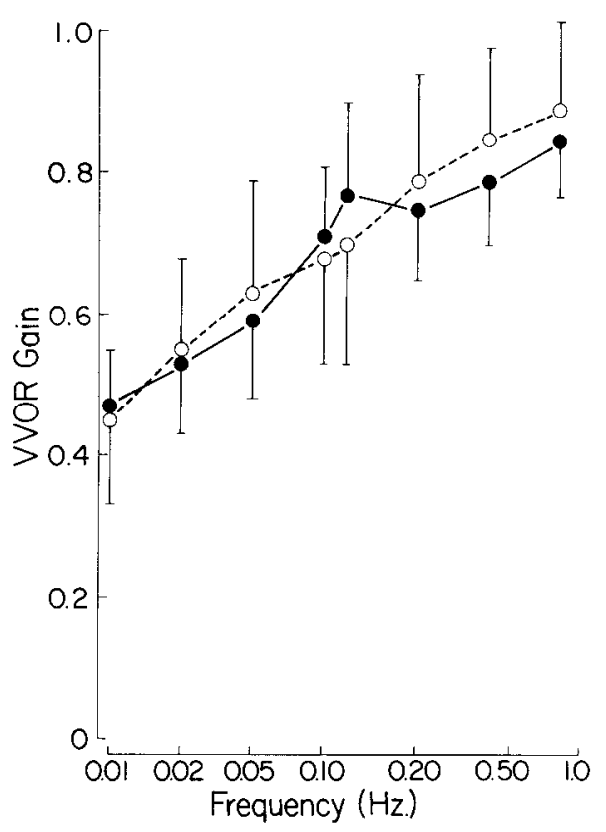

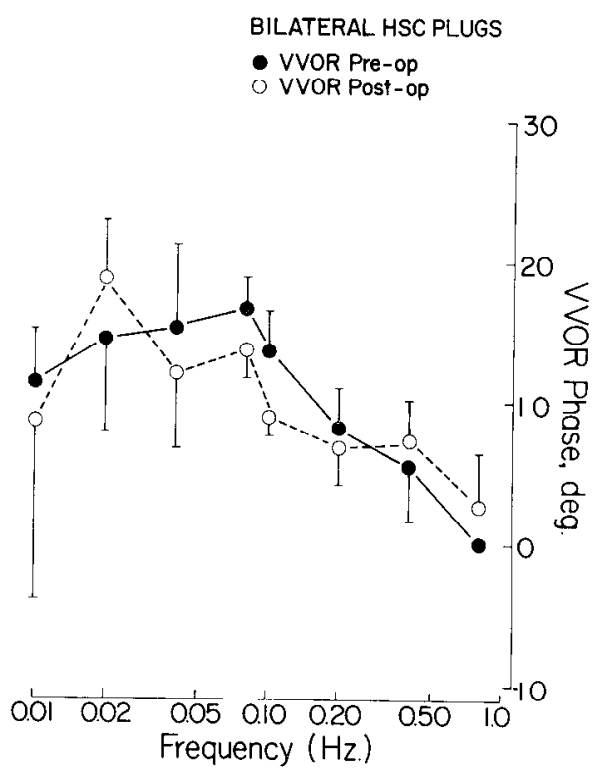

Figure 5. Influence of bilateral plugs of the HSCs on the VVOR. The VVOR of 5 rabbits was tested before (filled circles) and $24-48 \mathrm{hr}$ after (open circles) bilateral plugs were made in the HSCs. Note that these plugs did not influence the gain or phase of the VVOR. group of 5 rabbits that had received bilateral labyrinthectomies $10 \mathrm{~d}$ previously (Fig. 6). The VOKR was not tested in the canalplugged rabbits because, in a previous experiment, it was demonstrated that the gain of the VOKR in rabbits was not reduced by bilateral labyrinthectomies (Barmack et al., 1980).

\section{Effects of bilateral plugs of the anterior semicircular canals on the vertical and horizontal vestibuloocular reflexes and horizontal optokinetic reflex}

Bilateral plugs were placed in the ASCs of 7 rabbits for $24-48$ hr. The VVOR, HVOR, and HOKR were measured before the plugs were inserted, after they had been inserted, and at various times after the plugs were removed. Plugs of the ASCs caused a reduction in the gain of the VVOR at frequencies above 0.02 $\mathrm{H}_{7}$ (Fig. 7). After the plugs were removed, the recovery of the gain of the VVOR was followed over a period of $150 \mathrm{~d}$, at which time the gain of the VVOR had recovered to $92 \%$ of its preplug value (Fig. 7). Plugs of the ASCs caused a smaller change in the axes of eye movements than did plugs of the HSCs. Before the plugs were inserted, the eye movement orientation axes had an angle of $87.0^{\circ}$. This angle increased to $91.8^{\circ}$ when the ASCs were plugged. After the plugs were removed, the preplug axis angle of $87.0^{\circ}$ was reestablished within $4 \mathrm{~d}$. In addition to the reduction in the gain of the VVOR, bilateral plugs of the ASCs also caused a reduction in gain of the HVOR (Fig. 8). This reduction in gain was accompanied by an increased phase lead. After the plugs were removed, the gain and phase of the HVOR recovered to preplug values (Fig. 8).

The IIOKR was measured before plugs were inserted into the ASCs, when they were in place, and $150 \mathrm{~d}$ after the plugs were removed. Within the variability of the measurement technique, the gain of the HOKR was not reduced by the insertion of plugs into the ASCs (Fig. 9).

Time course of recovery of vestibuloocular reflexes following the removal of plugs from the horizontal and anterior semicircular canals

The recovery of vestibuloocular reflexes after removal of the semicircular canal plugs was measured at 2 different stimulus frequencies, 0.10 and $0.80 \mathrm{~Hz}$, after the plugs were removed. The recovery of the HVOR took approximately $10 \mathrm{~d}$ for the 0.8 $\mathrm{Hz}$ stimulus frequency (Fig. 10). At a stimulus frequency of 0.1 $\mathrm{Hz}$, the recovery was $88 \%$ complete at $15 \mathrm{~d}$. Longer recovery times were not evaluated in this group of rabbits. Since the VVOR was not impaired in rabbits with bilateral plugs of the HSCs when the plugs were in place, the VVOR was measured only at $0.40 \mathrm{~Hz}$ after the plugs were removed, in order to follow possible changes in the eye movement orientation axes.

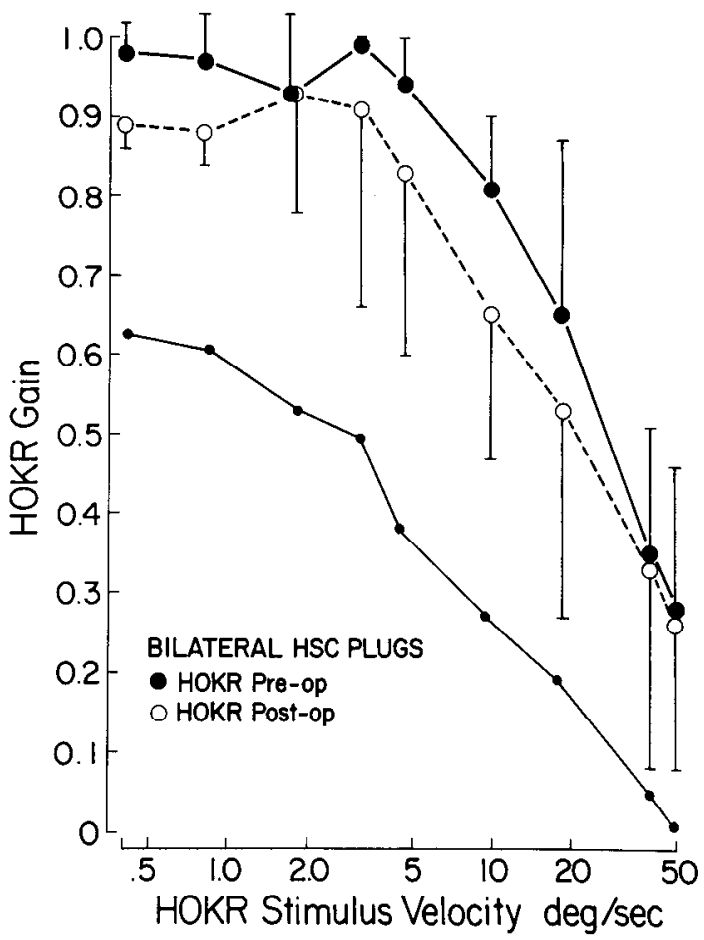

Figure 6. Influence of bilateral plugs of the HSCs on the HOKR. The HOKR was tested in 4 rabbits before ( filled circles) and 24-48 hr after (open circles) bilateral plugs of the HSCs. The HOKR was also tested in 5 rabbits after they had received bilateral labyrinthectomies (smaller filled circles). 
Figure 7. Influence of bilateral plugs of the ASCs on the VVOR. The VVOR of 7 rabbits was tested before ( filled circles), 24-48 hr after (open circles) the ASCs were plugged, and 5 months after the plugs were removed (filled triangles).

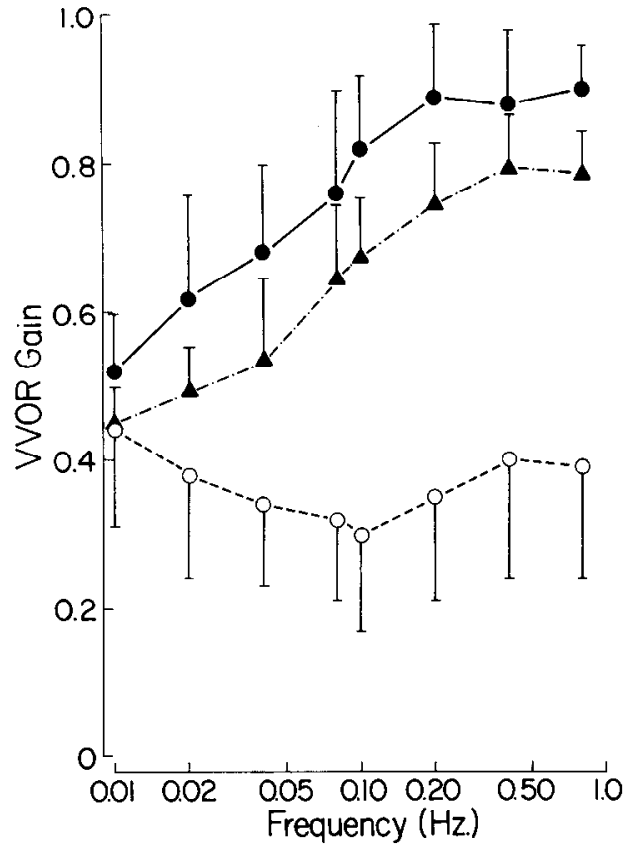

The recovery of the VVOR and HVOR following removal of plugs from the ASCs was measured over a period of $150 \mathrm{~d}$. The recovery of the VVOR was virtually complete after $15 \mathrm{~d}$, and there was only a nominal increase, observed after $150 \mathrm{~d}$, in the gain of the VVOR at a stimulus frequency of $0.1 \mathrm{~Hz}$ (Fig. 11). The recovery of the HVOR was complete within $10 \mathrm{~d}$ (Fig. 12).

Variations in the diameter of canal plugs and in the duration of the plug placement on the blockage and recovery of canal function

The HSCs of 3 rabbits were plugged with $75 \mu \mathrm{m}$ silver wires that had not been formed into spindles of larger diameter. These wires compressed the membranous semicircular canals, but did not completely occlude them. Nevertheless, the gain of the HVOR at $0.8 \mathrm{~Hz}$ was reduced from a mean of $0.85(\mathrm{SD}=0.15)$ before the plugs were inserted to a mean of $0.18(\mathrm{SD}=0.07)$ with the plugs in place. After the plugs were removed, the time course of recovery was similar to that of rabbits that had received more complete bilateral plugs with the larger spindles. In 2 rabbits, HSC plugs were inserted for just $1 \mathrm{~min}$ in each of the HSCs. The HVOR of these rabbits was measured $4 \mathrm{hr}$ after the operation at $0.8 \mathrm{~Hz}$. The gain of the HVOR was less than 0.3 for both rabbits, and the gain did not reach the preplug values of $0.8-0.9$ until $5 \mathrm{~d}$ after the operation.
Figure 8 . Influence of bilateral plugs of the ASCs on the HVOR. The HVOR of 7 rabbits was tested before (filled circles), 24-48 hr after (open circles) the ASCs were plugged, and $150 \mathrm{~d}$ after the plugs were removed (filled triangles).
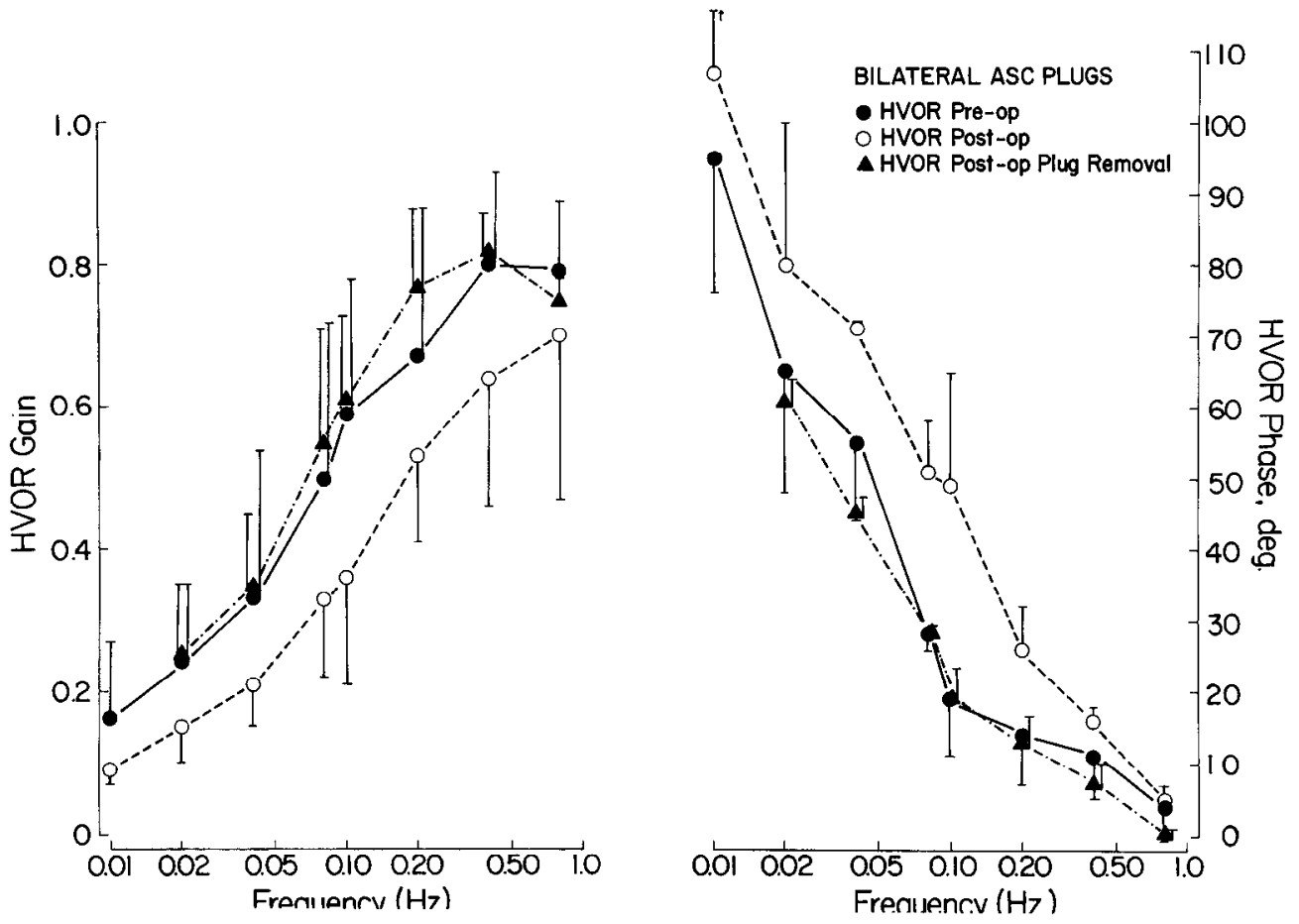


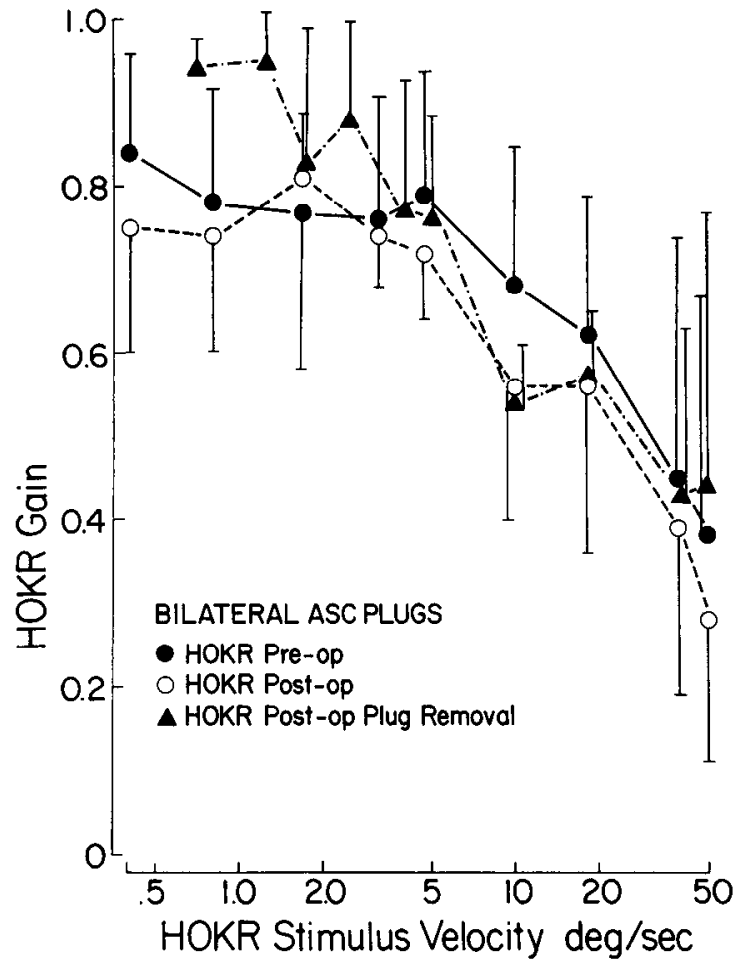

Figure 9. Influence of bilateral plugs of the ASCs on the HOKR. The HOKR was tested in 7 rabbits before (filled circles), 24-48 hr after (open circles) bilateral plugs were made in the ASCs, and $150 \mathrm{~d}$ after the plugs were removed (filled triangles).

\section{Histological analysis of plugged semicircular canals}

The labyrinths of 2 rabbits that had received unilateral plugs of the HSC were prepared for light-microscopic examination. The plugged labyrinths were compared with the unoperated labyrinths. One of the plugs had been left in place for $48 \mathrm{hr}$, and the other plug had been left in place for $30 \mathrm{~d}$. At the lightmicroscopic lcvel, no difference in the appearance of any of the plugged or unplugged labyrinths could be discerned (Fig. 13).

\section{Discussion}

There are several questions that may be asked with respect to the efficacy of the semicircular canal plugging procedure described in this experiment: Are plugged canals functionally blocked? Is the blockage restricted to the plugged canals? Does recovery from canal plugging indicate recovery of normal canal function or does it indicate a central adaptation or compensation for a permanently impaired peripheral vestibular apparatus? Is the plugging procedure reversible? These questions are addressed below.

\section{Are plugged semicircular canals functionally blocked?}

There is little doubt that the plugging procedure employed in these experiments functionally blocked the plugged semicircular canals by preventing changes in pressure across the cupula of the plugged canals by restricting the fluid dynamics of the endolymph. The evidence for this conclusion is most compelling from the data obtained with plugs of the HSCs. With the plugs in place, the gain of the HVOR was reduced to 0.00 at all frequencies below $0.1 \mathrm{~Hz}$, and was reduced to less than 0.05 at $0.8 \mathrm{~Hz}$ (Fig. 3). Consistent with this interpretation is the observation that plugs of the ASCs reversibly reduced the gain of

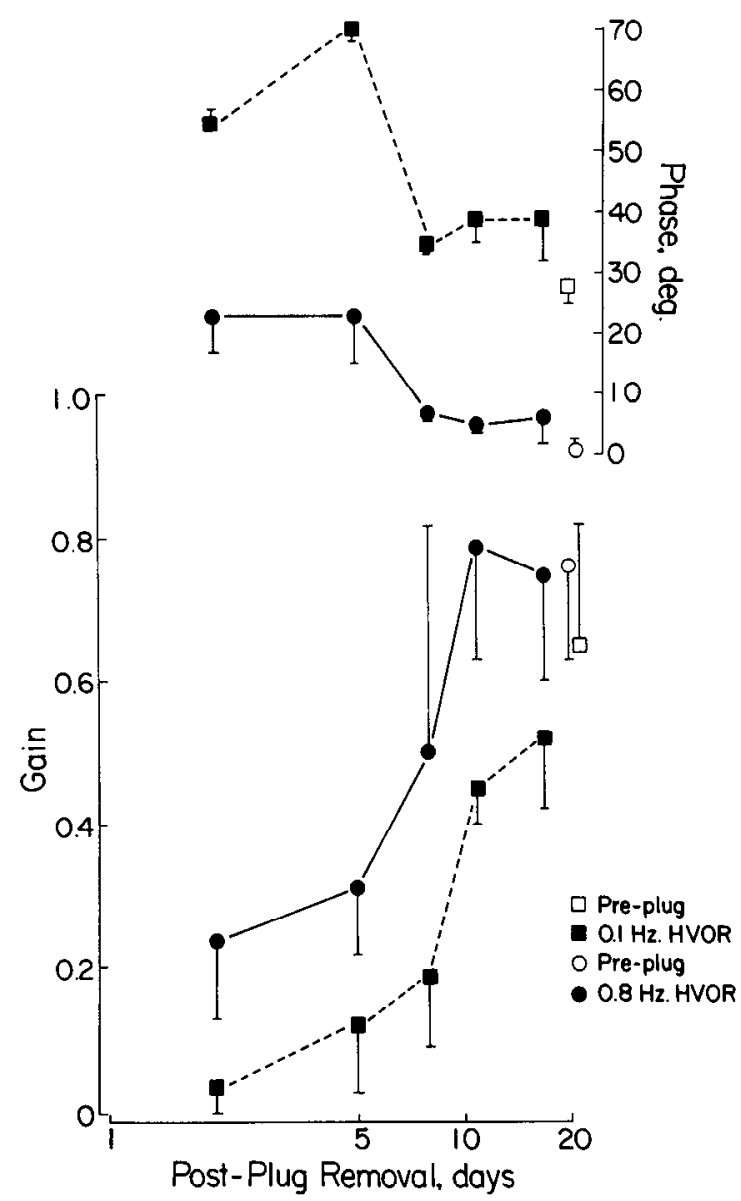

Figure 10. Recovery of the HVOR after the removal of bilateral plugs of the HSCs. The HVOR was tested in 5 rabbits at 2 different frequencies, $0.1 \mathrm{~Hz}$ (filled squares) and $0.8 \mathrm{~Hz}$ (filled circles) after removing the bilateral plugs from the HSCs. The preplug values for the gain and phase of the HVOR at these 2 frequencies are illustrated with open symbols. Note that the gain of the HVOR at $0.1 \mathrm{~Hz}$ recovered incompletely $15 \mathrm{~d}$ after the plugs were removed.

the HVOR by $10-40 \%$ in a frequency-dependent manner (Fig. 8). The changes in the orientation axes of the HVOR and VVOR following plugging of the HSCs, and the simple fact that the HSCs and ASCs are not truly orthogonal (Blanks et al., 1975; Curthoys, 1977; Barmack and Pettorossi, 1988a), also support the idea that the small residual HVOR originates from the unplugged ASCs.

The efficacy of plugs of the ASCs in reducing the gain of the VVOR is more difficult to evaluate, since the VVOR receives contributions from the posterior semicircular canals and the utricular otoliths as well as from the ASCs (Barmack and Pettorossi, 1988a). The VVOR in rabbits with plugged ASCs reflects the residual contributions of these other vestibular receptors (Barmack and Pettorossi, 1988a).

\section{Why do plugs of the ASCs reduce the gain of the HVOR?}

Although plugs of the HSCs did not reduce the gain of the VVOR, plugs of the ASCs did reduce the gain of the HVOR. These observations may be attributed, at least in part, to the fact that in the rabbit the semicircular canals are not truly orthogonal. The head orientation that would be optimal for stimulating one pair of semicircular canals (i.e., the HSCs) would not be optimal for minimizing the stimulation of a presumed 


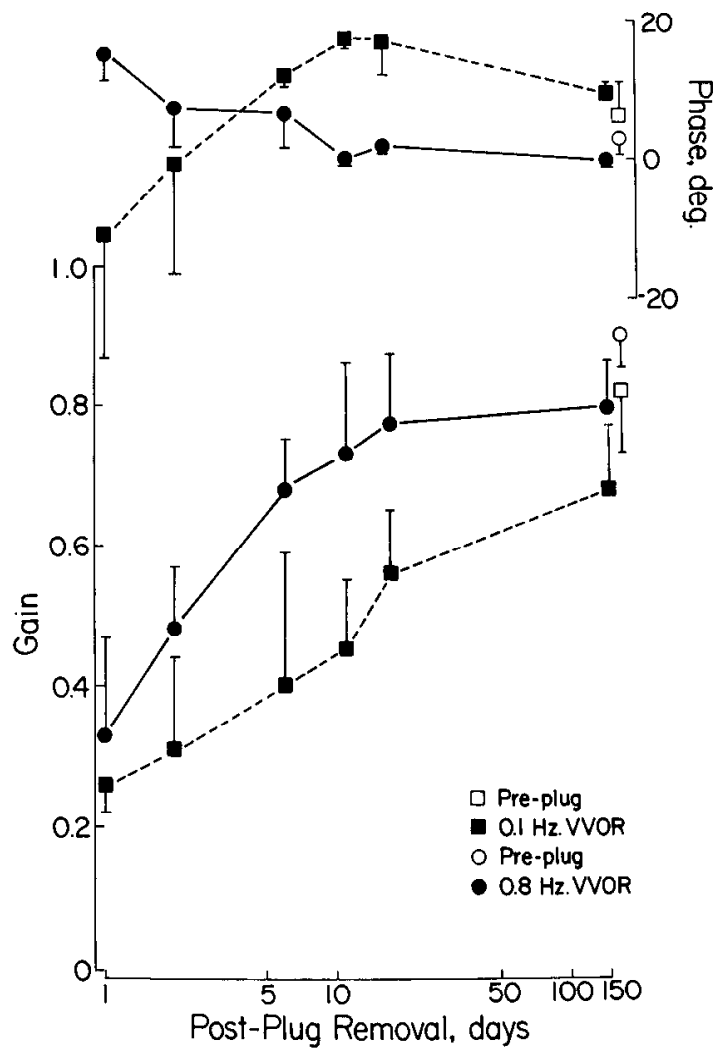

Figure 11. Recovery of the VVOR after the removal of bilateral plugs of the ASCs. The VVOR was tested in 7 rabbits at 2 different frequencies, $0.1 \mathrm{~Hz}$ (filled squares) and $0.8 \mathrm{~Hz}$ (filled circles) after removing the bilateral plugs from the ASCs. The preplug values for the gain and phase of the VVOR at these 2 frequencies are illustrated with open symbols.

orthogonal pair of semicircular canals (the ASCs) (Blanks et al., 1975; Curthoys et al., 1977; Baker et al., 1982). This might be considered additional evidence that the ASCs are coupled to the HVOR of the normal rabbit.

Does recovery from canal plugging indicate recovery of normal canal function or does it indicate a central adaptation or compensation for a permanently impaired peripheral vestibular apparatus?

One possible interpretation of the present data is that the recovery of the HVOR and VVOR following removal of the plugs from the HSCs and ASCs could be attributed to a central adaptation to a reduced peripheral signal rather than to a recovery of function of the peripheral labyrinth. This is unlikely. The gains of vestibuloocular reflexes in rabbits with permanent bilateral or unilateral plugged canals evince no signs of recovery even 1 month after the plugs are inserted (Barmack and Pettorossi, 1988a, b). The absence of histological evidence demonstrating peripheral damage leaves unanswered the question concerning what part of the peripheral labyrinth is damaged by the plugging operation.

\section{Is the plugging procedure reversible?}

The HOKR was not impaired by plugs of either the ASCs or the HSCs. The plugs were effective in functionally eliminating the contribution of the plugged canals to the HVOR or VVOR. One can infer from these findings only that the plugging operations did not significantly alter the level of spontaneous activity
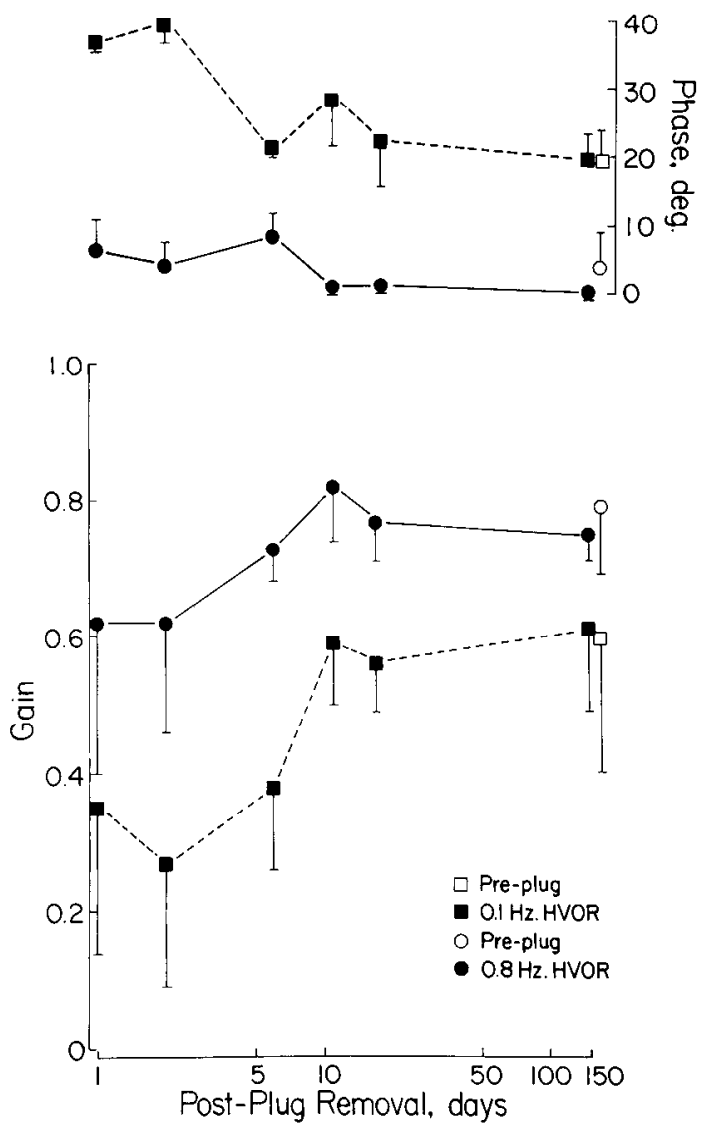

Figure 12. Recovery of the HVOR after the removal of bilateral plugs of the ASCs. The HVOR was tested in 7 rabbits at 2 different frequencies, $0.1 \mathrm{~Hz}$ (filled squares) and $0.8 \mathrm{~Hz}$ (filled circles) after removing the bilateral plugs from the ASCs. The preplug values for the gain and phase of the HVOR at these 2 frequencies are illustrated with open symbols. Note that complete recovery occurs within $15 \mathrm{~d}$.

of primary vestibular afferents. These findings are further supported by the observation that the present method of canal plugging, in contrast with previous methods, does not cause an immediate postoperative nystagmus when the plug is made unilaterally (Paige, 1983; Barmack and Pettorossi, 1988b). However, the recovery of the HVOR and VVOR from plugs of the HSCs and ASCs was not immediate even when the plugs were left in place for only $1 \mathrm{~min}$. Approximately 10-15 d after the plugs were removed, the recovery of the HVOR and VVOR was nearly complete. After this recovery period, there was a residual deficit of the HVOR at lower frequencies of vestibular stimulation amounting to approximately $10 \%$ of the preplug gain. This suggests that the plugging operation probably caused some damage to the membranous labyrinth or cupula, but that this damage was, for the most part, reversible. The present experiment could find no histological evidence that the plugging operation caused damage to the cristae ampulares of the plugged canals. However, similar negative results have been obtained for plugging operations performed using the bone dust-filling technique (Correia and Money, 1970). It is unlikely that the presumed damage caused by either method could be verified histologically, since the preparative techniques for histological analyses would subject the membranous labyrinth to pressures that were at least as traumatic as those to which the labyrinth was exposed during the plugging operation.

In sum, the plugging operations performed in this experiment 


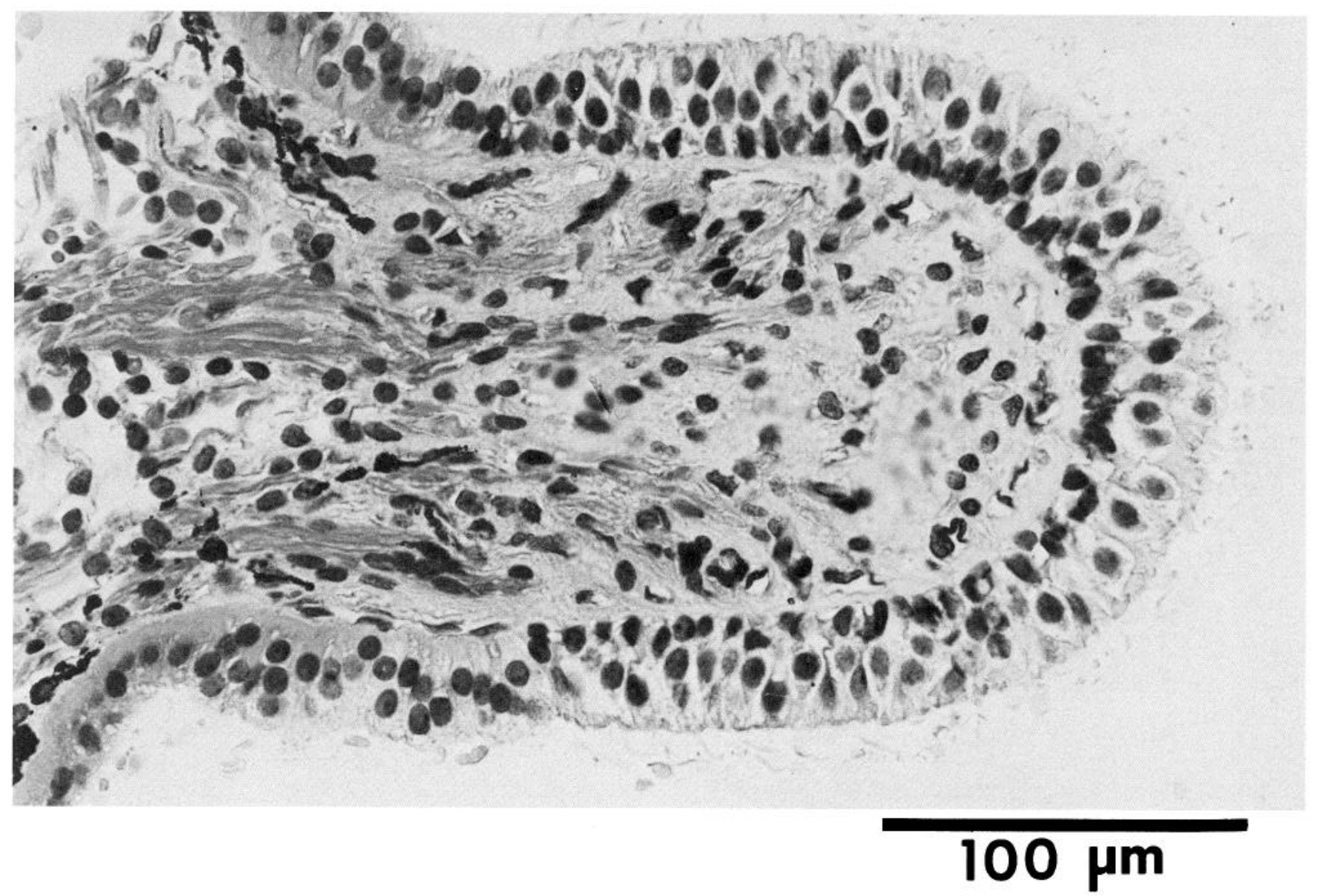

Figure 13. Light photomicrograph of an ampulla of a plugged HSC. The plug was left in position for $30 \mathrm{~d}$ before the rabbit was killed. There are no obvious losses of hair cells or other histological abnormalities detectable at the light-microscopic level.

were functionally reversible, but the time course of the recovery of function following the removal of plugs suggests that the operation does cause temporary damage to the labyrinth. These findings indicate that the technique of canal plugging would not be appropriate in acute experiments as a means of ascertaining the peripheral origins of a central vestibular response, but would be entirely appropriate for chronic experiments designed to test the contributions of different vestibular receptors to postural or vestibuloocular reflexes or as a method of examining the plasticity of vestibuloocular reflexes following unilateral blockage of semicircular canal function (Barmack and Pettorossi, 1988b).

\section{References}

Baarsma, E. A., and H. Collewijn (1975) Changes in compensatory eye movements after unilateral labyrinthectomy in the rabbit. Arch. Otorhinolaryngol. 211: 219-230.

Baker, J., J. Goldberg, B. Peterson, and R. Schor (1982) Oculomotor reflexes after semicircular canal plugging in cats. Brain Res. 252: 151155.

Barmack, N. H., and V. E. Pettorossi (1988a) The otolithic origin of the vertical vestibuloocular reflex following bilateral blockage of the vertical semicircular canals in the rabbit. J. Neurosci. 8: 2827-2835.

Barmack, N. H., and V. E. Pettorossi (1988b) The induction and compensation of asymmetric eye movements following unilateral blockage of a horizontal semicircular canal in the rabbit. J. Neurosci. 8: 2836-2843.

Barmack, N. H., V. E. Pettorossi, and R. G. Erickson (1980) The influence of bilateral labyrinthectomy on horizontal and vertical optokinetic reflexes in the rabbit. Brain Res. 196: 520-524.

Blanks, R. H. I., I. S. Curthoys, and C. H. Markham (1975) Planar relationships of the semicircular canals in man. Acta Otolaryngol. (Stockh.) 80: 185-196.

Bohmer, A., V. Henn, and J. I. Suzuki (1985) Vestibulo-ocular reflexes after selective plugging of the semicircular canals in the monkeyResponse plane determinations. Brain Res. 326: 291-298.

Capps, M. J., and J. A. Roth (1978) Visually evoked eye movements: Effects of labyrinthectomy. Exp. Neurol. 58: 251-260.

Cohen, B., T. Uemura, and S. Takemori (1973) Effects of labyrinthectomy on optokinetic nystagmus (OKN) and optokinetic afternystagmus (OKAN). Equilib. Res. 3: 88-93.

Collewijn, H. (1976) Impairment of optokinetic (after-)nystagmus by labyrinthectomy in the rabbit. Exp. Neurol. 52: 146-156.

Correia, M. J., and K. E. Money (1970) The effect of blockage of all six semicircular canal ducts on nystagmus produced by dynamic linear acceleration in the cat. Acta Otolaryngol. (Stockh.) 69: 7-16.

Curthoys, I. S., R. H. I. Blanks, and C. H. Markham (1977) Semicircular canal functional anatomy in cat, guinea pig and man. Acta Otolaryngol. (Stockh.) 83: 258-265.

Dow, R. S. (1938) The effects of unilateral and bilateral labyrinthectomy in monkey, baboon and chimpanzee. Am. J. Physiol. 121:392399.

Fisch, U. (1973) The vestibular response following unilateral vestibular neurectomy. Acta Otolaryngol. (Stockh.) 76: 229-238.

Goldberg, J. M., and C. Fernandez (1975) Responses of peripheral vestibular neurons to angular and linear accelerations in the squirrel monkey. Acta Otolaryngol. (Stockh.) 80: 101-110.

Henn, V., L. R. Young, and C. Finley (1974) Vestibular nucleus units in alert monkeys are also influenced by moving visual fields. Brain Res. 71: 144-149.

Jensen, D. W. (1979) Reflex control of acute postural asymmetry and compensatory symmetry after a unilateral vestibular lesion. Neuroscience 4: 1059-1073.

Keller, E. L., and P. D. Daniels (1975) Oculomotor related interaction 
of vestibular and visual stimulation in vestibular nucleus cells in alert monkey. Exp. Neurol. 46: 187-198.

Lacour, M., J. P. Roll, and M. Appaix (1976) Modifications and development of spinal reflexes in the alert baboon (Papio papio) following an unilateral vestibular neurotomy. Brain Res. 113: 255-269.

Maioli, C., W. Precht, and S. Ried (1983) Short- and long-term modifications of vestibulo-ocular response dynamics following unilateral vestibular nerve lesions in the cat. Exp. Brain Res. 50: 259-274.

Money, K. E., and J. W. Scott (1962) Functions of separate sensory receptors of nonauditory labyrinth of the cat. Am. J. Physiol. 202: 1211-1220.

Paige, G. D. (1983) Vestibuloocular reflex and its interactions with visual following mechanisms in the squirrel monkey. II. Response characteristics and plasticity following unilateral inactivation of horizontal canal. J. Neurophysiol. 49: 152-168.
Precht, W., H. Shimazu, and C. H. Markham (1966) A mechanism of central compensation of vestibular function following hemilabyrinthectomy. J. Neurophysiol. 29: 996-1010.

Rubin, A. M., S. C. R. Liedgren, A. C. Milne, J. A. Young, and J. M. Fredrickson (1977) Vestibular and somatosensory interaction in the cat vestibular nuclei. Pfluegers Arch. Physiol. 371: 155-160.

Seilly, D. J. (1982) A chemical test to determine the end point of EDTA decalcification. Med. Lab. Sci. 39: 71-73.

Suzuki, J. I., K. Goto, K. Tokumasu, and B. Cohen (1969) Implantation of electrodes near individual vestibular nerve branches in mammals. Ann. Otol. Rhinol. Laryngol. 78: 815-826.

Zee, D. S., R. D. Yee, and D. A. Robinson (1976) Optokinetic responses in labyrinthine-defective human beings. Brain Res. 113:423428. 